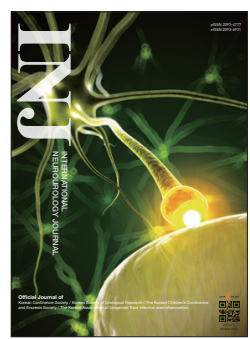

\title{
Chronic Pelvic Pain and Neuropelveology
}

\author{
Young Sam Cho (ib) https://orcid.org/0000-0002-2966-7971 \\ Department of Urology, Kangbuk Samsung Hospital, Sungkyunkwan University School of Medicine, Seoul, Korea \\ E-mail: choys1011@naver.com
}

The present issue of the International Neurourology Journal contains 2 interesting and important reviews. One is the "Neuropelveology: An Emerging Discipline for Management of Chronic Pelvic Pain" [1] and the other is "Current Pharmacologic Approaches in Painful Bladder Research - An Update” [2].

Patients with chronic pelvic pain (CPP) suffer from a silent ordeal', in that they often appear healthy, but experience unrelenting and intractable pain that requires frequent visits to hospitals for pain relief. Patients with CPP usually say that they just want to experience less pain, but it is often the case that such a desire does not work out well. Thus, physicians are very familiar with how these patients seek care at different hospitals and try various medications and treatment methods.

Much remains to be learned about CPP because the pathological processes underlying the condition have not yet been elucidated, biomarkers of CPP are not yet available, and the type and severity of symptoms can vary; thus, clearly defining the condition is not yet possible.

Interstitial cystitis/bladder pain syndrome (IC/BPS) is a heterogeneous syndrome that is usually characterized by urinary frequency, nocturia, and bladder pain [3]. In a case-control study in women with IC/BPS, the IC/BPS group had significant cognitive and psychosocial alterations compared to controls [4]. Irritable bowel syndrome was self-reported in $38.7 \%$ of patients versus $5.6 \%$ of controls, fibromyalgia was self-reported in $17.6 \%$ of patients versus $1.7 \%$ of controls, and chronic fatigue syndrome was self-reported in $9.4 \%$ of patients versus $1.1 \%$ of controls [4]. This means that IC/BPS patients do not all have the same cause and pathophysiology underlying their pelvic pain. What was originally considered a bladder disease is now con- sidered a chronic pain syndrome that may begin as a pathologic process in the bladder in most, but not all, patients.

From this point of view, neuropelveology is an appropriate term that reflects a very promising approach to these patients. Neuropelveology is an emerging paradigm focusing on the pelvic nervous system on a cross-disciplinary basis, including urology, gynecology, and neurology [1]. Possover et al. [1] suggested that the treatment of patients suffering from CPP requires more communication among neurology, the pelvic clinical disciplines, and basic research. Our current understanding of this highly variable syndrome does not favor the use of a monotherapy. In a collaborative and comprehensive review of 28 published randomized controlled trials, Magistro et al. [5] reported that the successful management of this condition is challenging and that a treatment strategy addressing the characteristics of individual patients is more effective than a single therapy.

On the basis of these efforts, research to find new treatment methods and to elucidate the pathophysiology should be continued. Andersson and Birder [2] reviewed current pharmacological approaches in painful bladder research. Inflammation and signaling from afferent nerves in the bladder and other pelvic organs play an important role in the symptoms of IC/BPS. Current research approaches including anti-nerve growth factor treatment and anti-tumor necrosis factor alpha treatment are potential systemic treatments, which implies that they may have effects both on the bladder and on nonbladder manifestations of IC/BPS.

The management of CPP requires a thorough and integrative knowledge of all pelvic organ systems and other systems, in- 
cluding the musculoskeletal, neurologic, and psychiatric systems. We expect that these various efforts and new concepts will be helpful to patients suffering from the ordeal of unrelenting pain due to CPP syndrome.

- Conflict of Interest: No potential conflict of interest relevant to this article was reported.

\section{REFERENCES}

1. Possover M, Andersson KE, Forman A. Neuropelveology: an emerging discipline for management of chronic pelvic pain. Int Neurourol J 2017;21:243-6.
2. Andersson KE, Birder L, Current pharmacologic approaches in painful bladder research: an up date. Int Neurourol J 2017;21:23542.

3. Jhang JF, Kuo HC. Pathomechanism of interstitial cystitis/bladder pain syndrome and mapping the heterogeneity of disease. Int Neurourol J 2016;20(Suppl 2):S95-104.

4. Nickel JC, Tripp DA, Pontari M, Moldwin R, Mayer R, Carr LK, et al. Psychosocial phenotyping in women with interstitial cystitis/ painful bladder syndrome: a case control study. J Urol 2010;183:16772.

5. Magistro G, Wagenlehner FM, Grabe M, Weidner W, Stief CG, Nickel JC. Contemporary Management of Chronic Prostatitis/ Chronic Pelvic Pain Syndrome. Eur Urol 2016;69:286-97. 